\title{
Mucopolysaccharidosis type I - Clinical and genetic characteristics of Romanian patients
}

\author{
Camelia Alkhzouz ${ }^{1,2 *}$, Cecilia Lazea ${ }^{1,2}$, Diana Miclea ${ }^{1,2}$, Carmen Asavoaie $^{2}$, Ioana \\ $\mathrm{Nascu}^{2}$, Tudor Pop ${ }^{1,2}$, Paula Grigorescu-Sido ${ }^{1,2}$
}

\author{
1. University of Medicine and Pharmacy "Iuliu Hatieganu” Cluj, Romania \\ Clinical Emergency Hospital for Children Cluj, Romania
}

\begin{abstract}
Background: Mucopolysaccharidosis type I (MPS I) is an autosomal recessive lysosomal storage disorder caused by a deficiency of $\alpha$-L-iduronidase (IDUA), which leads to the accumulation of partially digested glycosaminoglycans (dermatan sulfate and heparan sulfate) in the lysosomes and induces multisystemic alteration. Hurler (severe), Scheie (mild), and Hurler/Scheie (intermediate) syndromes are clinical subtypes of MPS-I. To date, more than 290 IDUA mutations have been reported. The purpose of this study was to present the clinical and genetic characteristics of Romanian MPS I syndrome patients and their genotype-phenotype correlation. Patients and methods: Seven patients (5 girls and 2 boys) with MPS type I, belonging to 4 unrelated families, aged 0,75-17.9 years, were enrolled. The study methods consisted in: clinical and standard auxological assessment, bone radiographs, joint ultrasonography, goniometry, neurological and psychological evaluation, hepatic and splenic ultrasonography, cardiological evaluation, otorhinolaryngology examination, ophthalmological examination, spirometry, $\alpha$-L-iduronidase enzyme activity assay and molecular analysis. Results: The seven patients originated from 4 unrelated families, three patients with severe, two patients with intermediate and two with attenuated clinical phenotype. Each patient presented the classical picture of MPS type I picture, represented by: variable coarse facial features, arthropathy, hepatosplenomegaly, cardiac involvement, respiratory dysfunction and neurological impairment. Five patological variants, three point mutations (p.Q70 *, p.I238Q and p.K324R), two deletion c.1045_1047delGAC, c.46_57delTCGCTCCTG) and an insertion (c.1389 insC) were identified in both alleles of the ADUA gene in homozygous or heterozygous form. Two novel mutations (p.K324R and c.1389 insC) were reported. The p. $Q 70^{*}(c .208 C>T)$ variant was identified in 2 families with severe form of disease (Hurler syndrome) in homozygous status in one family and in compound heterozygous status in the other family. Conclusion: The p.Q70* missense variant was the most frequent, correlated in all the cases who presented it with severe form, Hurler syndrome, the other mutations being usually isolated and particular for each patient, associated in our patients with less severe MPS I phenotype, as Hurler-Scheie or Scheie syndrome. The results of this study indicated the mutational heterogeneity of the IDUA gene and the difficulty to indicate some correlation between the genotype and phenotype in MPS I patients.
\end{abstract}

Keywords: MPS I, IDUA, mutations

Received: 12 ${ }^{\text {th }}$ April 2020; Accepted: $7^{\text {th }}$ July 2020; Published: $12^{\text {th }}$ July 2020

* Corresponding author: Camelia Alkhzouz, University of Medicine and Pharmacy "Iuliu Hatieganu” Cluj, Romania.E-mail: alkhzouz@yahoo.com 


\section{Introduction}

Mucopolysaccharidosis type I (MPS I) is a monogenic disease determined by $\alpha$-L-iduronidase (IDUA) deficiency. IDUA is a lysosomal enzyme involved in glycosaminoglycans (GAGs) degradation, IDUA deficiency having as consequence GAGs accumulations in different organs and tissues, thus leading to multisystemic involvement (1). The disease has three major clinical subtypes. Hurler syndrome is a severe subtype (MPS IH; 607014) characterized by infantile onset, frequent upper respiratory-tract infections, severe organomegaly, progressive skeletal dysplasia (dysostosis multiplex), reduction of linear growth and intellectual disability. The classical picture of intermediate Hurler-Scheie syndrome (MPS IH/S; 607015) with a clinical onset in childhood is represented by severe organomegaly, skeletal involvement and mild/moderate intellectual disability. Scheie syndrome (MPS IS; 607016) is less severe and is clinically characterized by: later onset, visceral and skeletal disease with progressive articular signs, cardiorespiratory involvement, with a psychomotor development which may be normal in early childhood (2). The gene encoding alpha-L-iduronidase (IDUA) has $19 \mathrm{~kb}$, is located on the chromosome 4p16.3 and contains 14 exons and 13 introns. It encodes a precursor IDUA protein of 653 amino acids, which is glycosylated and then processed to the mature form (3-5). The IDUA gene alterations include small gene alterations missense/nonsense, splicing, small deletions, small insertions, and large gene deletions, and complex rearrangements (Froissart et al. 1993). More than 290 distinct pathogenic variants of the IDUA gene have been described in the Human Genome Mutation Database (www. hgmdtrial.biobase-international.org 2019). Most of the identified mutations are point mutations $(52 \%)$ or small deletions $(17 \%)$. The two most common IDUA mutations reported in patients from Europe and North America are p.W402X and Q70X and their frequency are around 50\% of alleles (6-8). The other part of patients are carrying "private" mutations $(7,9)$. Genetic testing of the MPS I patients is useful for the identification of specific genotypes, to indicate genotype-phenotype correlations and also to indicate a correct reproductive option in prenatal diagnosis.

The aim of this research was to study the phenotype and genotype features of the Romanian patients with MPS I syndrome.

\section{Patients and methods}

In this study we investigated seven patients with MPS type I, five girls and two boys. They belonged to four unrelated families and their age at diagnosis was between 0.75 and 17.9 years. The enrolment period for this study was between 2005 and 2018 and these patients represent all the patients diagnosed in Romania within this time interval. The study protocol had the approval of the Ethics Committee of Clinical Emergency HospitalforChildren,Cluj-Napoca(no.692/date27.01. 2014). For each patient a written and informed consent to participate at this study was obtained from their parents or legal guardians (10).

The patients were clinically evaluated including the following: auxologic measurements (using www.who.int/childgrowth/standards, Seca Vogel and Halke Hamburg 702 device), goniometry, radiology (skeletal assessment), ultrasonographic, neurological, psychological, otorhinolaryngological (including audiogram), ophthalmological, cardiological (ECG, Doppler echocardiography) evaluations and spirometry. Ultrasonographic liver and spleen volumes were expressed as multiple of normal value, calculated for each subject function of their weight, using the accepted cut-off values of $2.5 \%$ of their body weight for the liver respectively of $0.2 \%$ of their body weight for the spleen $(11,12)$. The diagnosis was specifically determined for each 
subject using an enzymatic and genetic assessment. The enzymatic activity of $\alpha$-L-iduronidase was evaluated using the analyse of this activity in plasma (Biochemistry Department of „Iuliu Hatieganu" University of Medicine and Pharmacy, Cluj-Napoca) or the fluorometric determination of 4-methylumbelliferone using dried blood spot (Centogene Laboratory Rostock, Hamburg). These two different methods were done according to the possibilities that we had at different periods of time. Genetic testing was done respecting the ethical standards of the hospital committee, according to Helsinki Declaration of 1975 (revised in 2000). Genetic evaluation was performed at Archimed Life Science GnbH Laboratory, Vienna and Centogene Laboratory, Rostock. The genetic analysis of IDUA gene consisted in sequencing of all exons and intronic regions using NM_000203.3. Novel as reference sequence for IDUA gene. Polyphen-2 platform was used to predict the pathogenic effect of unreported mutations.

\section{Results}

The seven patients originated from 4 unrelated families, three patients/2 families (F3, F4) with severe, Hurler syndrome, two patients (F1) with intermediate, Hurler-Scheie syndrome and two with attenuated (F2) clinical phenotype, Scheie syndrome. The specific diagnosis in all 3 families with two affected children was established only after the birth of the second child, although the clinical picture in the first child was strongly suggestive for this disease.

The clinical features of the seven patients with MPS I are presented in Table I.

The height presented important variations, between -0.6 SD and -7.8 SD, depending on age, therefore the mean height was determined in two groups: in patients under 7 years, which presented $-2.42 \mathrm{SD}$, respectively $-6.25 \mathrm{SD}$ mean height in patients aged between 7 and 17.9 years. The height was normal in the first years, then the growth velocity progressively decreases with age. In each patient was observed the classical picture of MPS type 1 syndrome: coarse facial features, clinical signs of articular and skeletal involvement with stiff joints, umbilical and/or inguinal hernia and hepatosplenomegaly. The mean volume of the liver was $2.03 \pm 1.57$ multiple of normal liver volume (calculated for each subject function of his weight) and the mean spleen volume was $3.65 \pm 1.78$ multiple of normal spleen volume (calculated for each subject function of his weight). Concerning the cardiac involvement, it was represented by variable degrees of left ventricular hypertrophy and valvopathies. All the patients presented mitral valve thickening with different degrees of regurgitation, depending on the age at diagnosis. Aortic insufficiency was observed in four patients. Corneal opacities in variable degree were present in five patients. Neurological and psychological assessment revealed 2 siblings with normal intellectual development and 5 patients with a variable neuro-intellectual disability, from mild deficit to severe. The mean age of clinical onset was $1.17 \pm 1.94$ years; the clinical diagnosis was established at the age of $6.62 \pm 7.26$ years, and specific genetic diagnosis was established at $7.74 \pm 7.12$ years. It was observed an important delay ( $3.4 \pm 2.36$ years) between the age at clinical onset to the moment of specific genetic diagnosis (the limits: 1 month and 12.7 years). A Scheie syndrome patient (the younger sister, F3) late diagnosed, died shortly after confirmation of the diagnosis prior to initiation of enzyme replacement therapy, due to severe heart disease. The enzyme replacement therapy was started at mean the age of $6.92 \pm 9.09$ years (limits: $1.75-$ 19 years), the average duration of therapy was $6.37 \pm 5.37$ years with a range between 0.25 and 13 years. The $\alpha$-L-iduronidase activity presented a markedly reduction or absence in all patients (Table II.). 


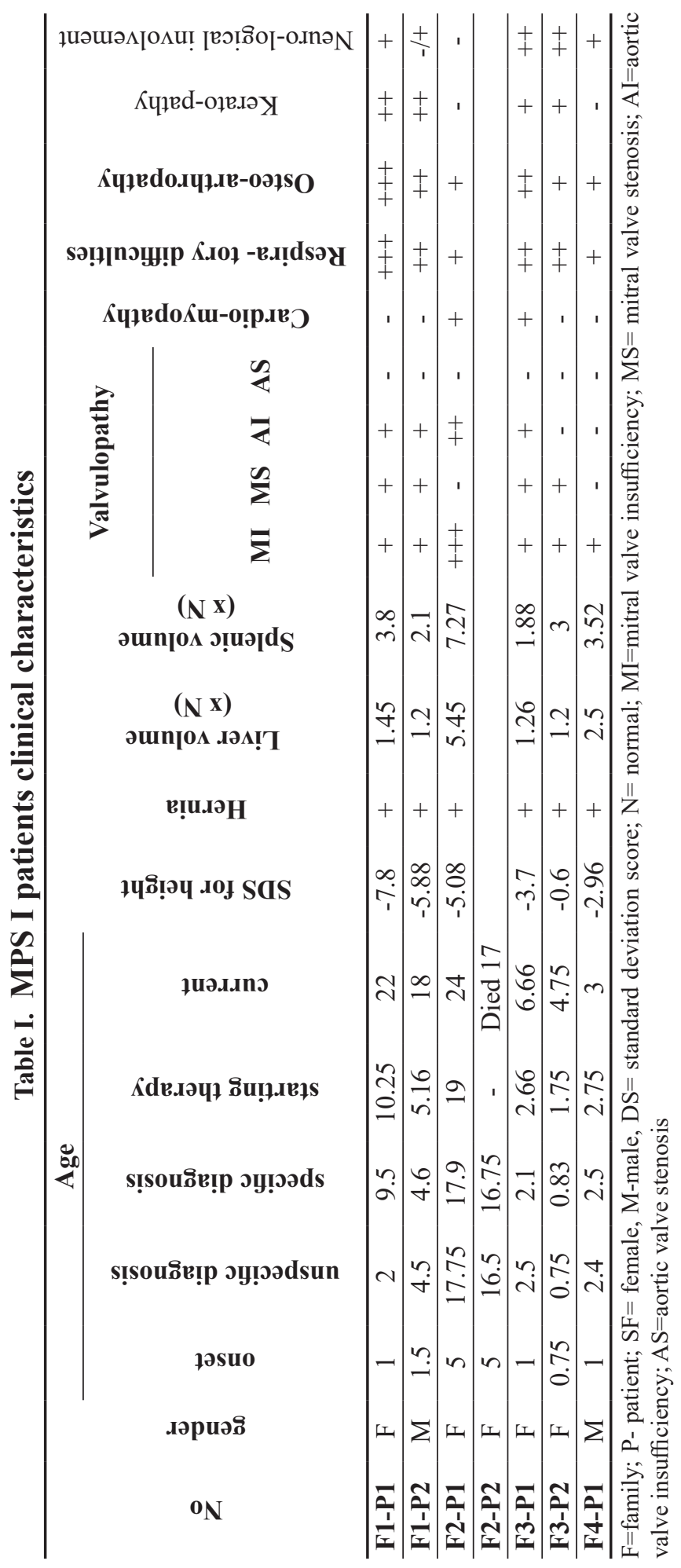

The molecular analysis identified six different diseases causing pathological variants, 3 point mutations ( 1 nonsense and 2 missense mutations), 2 deletions, 1 insertions and 2 polymorphic sites (SNP) mutation (Table III). We found homozygosity in two siblings (F3), whereas the other five patients (F1, F2, F4) showed the presence of compound heterozygous IDUA genotype. Two of these were novel pathogenic variants.

In two sisters (F3) with severe phenotype (Hurler syndrome) was detected the c. $208 \mathrm{C}>\mathrm{T}$ p.Q70* homozygous mutation in exon 2 and a second homozygous mutation c. $352 \mathrm{C}>\mathrm{T}$ p.L118L in exon 3 - SNP.

In a $\mathrm{F} 4$ patient, another patient with Hurler syndrome, it was also detected the c.208C $>\mathrm{T}$ p.Q70* pathogenic variant in exon 2 and the second, c.46_57delTCGCTCCTG, p.S16 A19delGCC, in exon 1.

In two siblings with Hurler-Scheie syndrome (F1) two unreported mutations were identified: the c. $971 \mathrm{~A}>\mathrm{G}$ results in a missense mutation p.K324R which is predicted to be probably damaging with a score of 1.000 by Polyhen-2 and an insertion of one base pair in exon 9 resulting in frameshift and premature stop.

In F2 family with Scheie syndrome two mutations were detected: c.713T $>$ A missens mutation p.L238Q and a deletion c.1045_1047delGAC.

The most frequent mutation in our group was the p.Q70*.

\section{Discussion}

In this study we described the clinical and molecular characterization of the MPS type I patients, being the first one on Romanian patients. The seven patients originated from 4 unrelated families, three patients/2 families (F3, F4) with severe, two patients (F1) with 
Table II. $\alpha$-L-Iduronase activity

\begin{tabular}{ccccc}
\hline No. & Enzyme activity & Normal range & Unit of measure & Sample type \\
\hline F1-P1 & 0 & $300-800$ & $\mathrm{mMol} / \mathrm{l} / \mathrm{h}$ & Plasma \\
\hline F1-P2 & 0 & $300-800$ & $\mathrm{mMol} / \mathrm{l} / \mathrm{h}$ & Plasma \\
\hline F2-P1 & 0 & $300-800$ & $\mathrm{mMol} / \mathrm{l} / 4 \mathrm{~h}$ & Plasma \\
\hline F2-P2 & 0 & $300-800$ & $\mathrm{mMol} / \mathrm{l} / 4 \mathrm{~h}$ & Plasma \\
\hline F3-P1 & $<0.5$ & $\geq 2$ & $\mu \mathrm{mol} / \mathrm{l} / \mathrm{h}$ & $\mathrm{DBS}$ \\
\hline F3-P2 & $<0.5$ & $\geq 2$ & $\mu \mathrm{mol} / \mathrm{h} / \mathrm{h}$ & $\mathrm{DBS}$ \\
\hline F4-P1 & $<0 . .5$ & $\geq 2$ & $\mu \mathrm{mol} / \mathrm{h}$ & $\mathrm{DBS}$ \\
\hline
\end{tabular}

$\mathrm{F}=$ Family; $\mathrm{P}=$ Patient; $\mathrm{DBS}=$ dried blood spot

Table III. MPS I patients molecular characteristics

\begin{tabular}{|c|c|c|c|c|c|c|c|}
\hline Nr. & $\begin{array}{l}\text { Al- } \\
\text { lele }\end{array}$ & Location & $\begin{array}{l}\text { Nucleotide } \\
\text { change }\end{array}$ & $\begin{array}{c}\text { Amino } \\
\text { acid } \\
\text { change }\end{array}$ & Reference & $\begin{array}{c}\text { Biochemical } \\
\text { phenotype }\end{array}$ & $\begin{array}{l}\text { Clinical } \\
\text { form }\end{array}$ \\
\hline \multirow{2}{*}{$\mathrm{F} 1$} & 1 & Exon 8 & c. $971 \mathrm{~A}>\mathrm{G}$ & p.K324R & - & \multirow{2}{*}{ no enzyme activity } & \multirow{2}{*}{$\mathrm{H}-\mathrm{S}$} \\
\hline & 2 & Exon 9 & c. 1389 ins $\mathrm{C}$ & - & - & & \\
\hline \multirow{2}{*}{$\mathrm{F} 2$} & 1 & Exon 7 & c. $713 \mathrm{~T}>\mathrm{A}$ & p.L238Q & Yogalinga, 2004 & \multirow{2}{*}{ no enzyme activity } & \multirow{2}{*}{ S } \\
\hline & 2 & Exon 8 & c.1045_1047delGAC & & Bertola, 2011 & & \\
\hline \multirow{4}{*}{ F3 } & 1 & Exon 2 & c. $208 \mathrm{C}>\mathrm{T}$ & p.Q70* & Clarke, 1993 & \multirow{4}{*}{$\begin{array}{l}\text { low enzyme } \\
\text { activity }\end{array}$} & \multirow{4}{*}{$\mathrm{H}$} \\
\hline & 1 & Exon 3 & c. $352 \mathrm{C}>\mathrm{T}$ & p.L118L & rs37559954 & & \\
\hline & & Exon 2 & c. $208 \mathrm{C}>\mathrm{T}$ & p.Q70* & Clarke, 1993 & & \\
\hline & 2 & Exon 3 & c. $352 \mathrm{C}>\mathrm{T}$ & p.L118L & rs37559954 & & \\
\hline \multirow[b]{2}{*}{$\mathrm{F} 4$} & 1 & Exon 2 & c. $208 \mathrm{C}>\mathrm{T}$ & p.Q70* & Clarke 1993 & \multirow{2}{*}{$\begin{array}{l}\text { low enzyme } \\
\text { activity }\end{array}$} & \multirow[b]{2}{*}{$\mathrm{H}$} \\
\hline & 2 & Exon 1 & $\begin{array}{c}\text { c.46_57delTC- } \\
\text { GCTCCTGGCC }\end{array}$ & $\begin{array}{l}\text { p.S16- } \\
\text { A19del }\end{array}$ & Bunge, 1993 & & \\
\hline
\end{tabular}

$\mathrm{F}=$ family; $\mathrm{SNP}=$ single nucleotide polymorphism; H-Hurler syndrome; $\mathrm{S}=$ Scheie syndrome; $\mathrm{H}-\mathrm{S}=$ Hurler=Scheie syndrome

intermediate and two with attenuated (F2) clinical phenotype. Each patient presented the classical picture of MPS type I picture, represented by: variable coarse facial features, arthropathy, hepatosplenomegaly, cardiac involvement, respiratory dysfunction and neurological impairment.

The p.Q70*(c.208C $>$ T) variant was identified in 2 families with severe form of disease (Hurler syndrome) in homozygous status in one family (F3) and in compound heterozygous status in the other family (F4). The p.Q70*(c.208C > T) variant induce a stop codon, predicted to prematurely end the transcript (9). Biochemical assays already described for these variants showed essentially no activity in homozygotes (13), concord- ant with Hurler phenotype, in our patients being observed a very low activity for the enzyme, the clinical phenotype being also Hurler syndrome. The incidence of p.Q70* (c.208C $>$ T) mutation, $3 / 8$ alleles of pathological variants of IDUA gene alleles identified in our group is according to results reported by other studies $(5,14-19)$. In F4, compound heterozygous p.Q70*(c.208C > T), the second variant is a deletion located on exon 1 (p.S16_A19del, 46_57delTCGCTCCTGGCC), described by Bunge in 1994, as a mutation that induces a severe phenotype $(3,20)$. The phenotype for this patient, as for the siblings of F3 family, homozygous for p.Q70* (c.208C > T) was also severe, as Hurler syndrome. 
In two siblings (F1) with intermediate form, Hunter-Scheie syndrome, two new mutations were identified: c.971A $>$ G (p.K324R), which leads to a missense variant (undescribed variant at the moment of determination, in 2015, but subsequently described by Uttarilli et al in 2016 in a patient from India, with low enzyme activity) (21); and an insertion of one base pair in exon 9 resulting in frameshift and premature stop (c.1389 ins C) (not described until now). The variable expression of the different variants of IDUA gene is indicated by the large spectrum of biochemical and clinical signs (22).

In F2, two mutations were detected: c.713T $>\mathrm{A}$ results in a known missense mutation p.L238Q, which described reduced IDUA protein and activity level, as in our patient (23) and a deletion of 3 base pair in exon 10 (c.1045_1047delGAC), also associated with low enzymatic level (8). The patient from F2 family presented the less severe MPS I phenotype, Scheie syndrome.

Overall, most mutations were "private", with only one mutation (Q70*) being common in our group, according to other studies $(8,24)$.

Our results confirm the degree of variants heterogeneity observed in MPS I, in concordance with other previous studies $(25,26)$. This allelic heterogeneity does not permit to establish clear correlations between mutant genotypes and phenotypes (27). A limitation for our study is the limited number of patients to allow clear conclusions regarding the phenotype genotype correlations in our patients.

\section{Conclusions}

The p.Q70* missense variant was the most frequent, correlated in all the cases who presented it with severe form, Hurler syndrome, the other mutations being usually isolated and particular for each patient, associated in our patients with less severe MPS I phenotype, as Hurler-Scheie or Scheie syndrome. The results of this study indicated the mutational heterogeneity of the IDUA gene and the difficulty to indicate some correlation between the genotype and phenotype in MPS I patients.
Abbreviations
MPS I - Mucopolysaccharidosis type I
IDUA - $\alpha$-L-iduronidase
GAGs - glycosaminoglycans
$\mathrm{H}$ - Hurler syndrome
H-S - Hurler-Scheie syndrome
$\mathrm{S}$ - Scheie syndrome
F - family
SF - female
$\mathrm{M}$ - male
DS - standard deviation score
$\mathrm{N}$ - normal
MI - mitral valve insufficiency
MS - mitral valve stenosis
AI - aortic valve insufficiency
AS - aortic valve stenosis
DBS - dried blood spot
SNP - single nucleotide polymorphism.

\section{Authors' contribution}

CAl (conceptualization, methodology, validation, investigation, manuscript writing, manuscript supervising)

CL (methodology, validation, investigation)

DM (methodology, manuscript writing)

CAs (methodology, validation, investigation)

IN (methodology, validation, investigation)

TP (methodology, validation, investigation)

PGS (conceptualization, methodology, validation, investigation)

\section{Conflict of interest}

None to declare. 


\section{References}

1. The mucopolysaccharidoses. The metabolic and molecular bases of inherited disease; [Internet]. 2001.

2. D'Aco K, Underhill L, Rangachari L, Arn P, Cox GF, Giugliani R, et al. Diagnosis and treatment trends in mucopolysaccharidosis I: findings from the MPS I Registry. Eur J Pediatr. 2012;171(6):911-9.

3. Scott HS, Guo X-H, Hopwood JJ, Morris CP. Structure and sequence of the human $\alpha$-L-iduronidase gene. Genomics. 1992;13(4):1311-3.

4. Taylor J, Gibson G, Brooks D, Hopwood J. $\alpha$-1-Iduronidase in normal and mucopolysaccharidosis-type-I human skin fibroblasts. Bioch J. 1991;274 ( Pt 1):263-8.

5. Scott HS, Bunge S, Gal A, Clarke LA, Morris CP, Hopwood JJ. Molecular genetics of mucopolysaccharidosis type I: diagnostic, clinical, and biological implications. Hum Mutat. 1995;6(4):288-302.

6. Brooks D. The immunochemical analysis of enzyme from mucopolysaccharidoses patients. JIMD. 1993;16(1):3-15.

7. Scott HS, Litjens T, Nelson PV, Brooks DA, Hopwood JJ, Morris CP. $\alpha$-L-Iduronidase mutations (Q70X and P533R) associate with a severe Hurler phenotype. Hum Mutat. 1992;1(4):333-9.

8. Bertola F, Filocamo M, Casati G, Mort M, Rosano C, Tylki-Szymanska A, et al. IDUA mutational profiling of a cohort of 102 European patients with mucopolysaccharidosis type I: identification and characterization of 35 novel $\alpha$-L-iduronidase (IDUA) alleles. Hum $\mathrm{Mu}$ tat. 2011;32(6):E2189-210.

9. Clarke LA, Nelson PV, Warrington CL, Morris CP, Hopwood JJ, Scott HS. Mutation analysis of 19 North American mucopolysaccharidosis type I patients: identification of two additional frequent mutations. Hum Mutat. 1994;3(3):275-82.

10. Jurca C, Bembea M, Pallag A, Muresan M, Szilagyi A, Balmos A, et al. Pharmacotherapeutical considerations in the treatment and management of neonatal hyperammonaemia. Farmacia. 2018;66(2):216-22.

11. Patlas M, Hadas-Halpern I, Abrahamov A, Zimran A, Elstein D. Repeat abdominal ultrasound evaluation of 100 patients with type I Gaucher disease treated with enzyme replacement therapy for up to 7 years. Hematol J. 2002;3(1):17-20.

12. Weinreb NJ, Charrow J, Andersson HC, Kaplan P, Kolodny EH, Mistry P, et al. Effectiveness of enzyme replacement therapy in 1028 patients with type 1 Gaucher disease after 2 to 5 years of treatment: a report from the Gaucher Registry. Am J Med. 2002;113(2):112-9.

13. Wang D, Shukla C, Liu X, Schoeb TR, Clarke LA, Bedwell DM, et al. Characterization of an MPS IH knockin mouse that carries a nonsense mutation analogous to the human IDUA-W402X mutation. Mol Genet Metab. 2010;99(1):62-71.
14. Taghikhani M, Khatami S, Abdi M, Hakhamaneshi MS, Alaei MR, Zamanfar.D J. Mutation analysis and clinical characterization of Iranian patients with mucopolysaccharidosis type I. Clin Lab Anal. 2019;00:e22963

15. Zahoor MY, Cheema HA, Ijaz S, Anjum MN, Ramzan $\mathrm{K}$, Bhinder MA. Mapping of IDUA gene variants in Pakistani patients with mucopolysaccharidosis type 1 . J Pediatr Endocrinol Metab. 2019;32(11):1221-1227.

16. Clarke LA, Giugliani R, Guffon N, Jones SA, Keenan HA, Munoz-Rojas MV et al. Genotype-phenotype relationships in mucopolysaccharidosis type I (MPS I): Insights from the International MPS I Registry. Clin Genet. 2019;96(4):281-289.

17. Zanetti A, D’Avanzo F, Rigon L, Rampazzo A, Concolino D, Barone R, et al. Molecular diagnosis of patients affected by mucopolysaccharidosis: a multicenter study. Eur J Pediatr. 2019;178(5):739-753.

18. Beesley CE, Meaney CA, Greenland G, Adams V, Vellodi A, Young EP, et al. Mutational analysis of 85 mucopolysaccharidosis type I families: frequency of known mutations, identification of 17 novel mutations and in vitro expression of missense mutations. Hum Genet. 2001;109(5):503-11.

19. Pollard LM, Jones JR, Wood TC. Molecular characterization of 355 mucopolysaccharidosis patients reveals 104 novel mutations. JIMD. 2013;36(2):179-87.

20. Bunge S, Kleijer WJ, Steglich C, Beck M, Zuther C, Morris CP, et al. Mucopolysaccharidosis type I: identification of 8 novel mutations and determination of the frequency of the two common $\alpha$-L-iduronidase mutations (W402X and Q70X) among European patients. Hum Mol Genet. 1994;3(6):861-6.

21. Uttarilli A, Ranganath P, Matta D, Md Nurul Jain J, Prasad K, Babu AS, et al. Identification and characterization of 20 novel pathogenic variants in 60 unrelated Indian patients with mucopolysaccharidoses type I and type II. Clin Genet. 2016;90(6):496-508.

22. Terlato NJ, Cox GF. Can mucopolysaccharidosis type I disease severity be predicted based on a patient's genotype? A comprehensive review of the literature. Genet Med. 2003;5(4):286-94.

23. Yogalingam G, Guo XH, Muller V, Brooks DA, Clements P, Kakkis E, et al. Identification and molecular characterization of $\alpha$-L-iduronidase mutations present in mucopolysaccharidosis type I patients undergoing enzyme replacement therapy. Hum Mutat. 2004;24(3):199-207.

24. Gatti R, DiNatale P, Villani G, Filocamo M, Muller V, Guo X-H, et al. Mutations among Italian mucopolysaccharidosis type I patients. Journal of inherited metabolic disease. 1997;20(6):803-6.

25. Gort L, Chabás A, Coll MJ. Analysis of five mutations in 20 mucopolysaccharidosis type I patients: high prevalence of the W402X mutation. Hum Mutat. 1998;11(4):332-3. 
26. Matte U, Yogalingam G, Brooks D, Leistner S, Schwartz I, Lima L, et al. Identification and characterization of 13 new mutations in mucopolysaccharidosis type I patients. Mol Genet Metab. 2003;78(1):37-43.
27. Li P, Wood T, Thompson JN. Diversity of mutations and distribution of single nucleotide polymorphic alleles in the human $\alpha$-1-iduronidase (IDUA) gene. Genet Med. 2002;4(6):420. 